\title{
Epidemiology of anti-tuberculosis drug resistance patterns and trends in tuberculosis referral hospital in Addis Ababa, Ethiopia
}

Dereje Abate ${ }^{1,2}$, Bineyam Taye ${ }^{1,3^{*}}$, Mohammed Abseno $^{2}$ and Sibhatu Biadgilign ${ }^{4}$

\begin{abstract}
Background: Drug-resistant TB has emerged as a major challenge facing TB prevention and control efforts. In Ethiopia, the extent/trend of drug resistance TB is not well known. The aim of this study was to determine the pattern and trend of resistance to first line anti-TB drugs among culture positive retreatment cases at St.Peter's TB Specialized Hospital.
\end{abstract}

Findings: A hospital based retrospective study was used to assess the pattern of anti-TB drug resistance among previously treated TB patients referred to St.Peter's TB Specialized Hospital from January 2004-December 2008 Gregorian calendar(GC) for better diagnosis and treatment.

Among 376 culture positive for M. tuberculosis one hundred and two (27.1\%) were susceptible to all of the four first line anti-TB drugs -Isoniazid (INH), Rifampicin (RIF), Ethambutol (ETB) \& Streptomycin (STM). While 274 (72.9\%) were resistant to at least one drug. Any resistance to STM (67.3\%) was found to be the most common and the prevalence of MDR-TB was 174 (46.3\%). Trend in resistance rate among re-treatment cases from 2004 to 2008 showed a significant increase for any drug as well as for INH, RIF, and MDR resistance ( $P<0.05$ for trend).

Conclusions: There has been an increasing trend in drug resistance in recent years, particularly in retreatment cases. Therefore, establishing advanced diagnostic facilities for early detection of MDR-TB and expanding second line treatment center to treat MDR-TB patients and to prevent its transmission is recommended.

Keywords: Epidemiology, MDR-TB, Drug resistance, Drug susceptibility, Trend

\section{Introduction}

Tuberculosis (TB) is a disease impacting public health problem worldwide that disproportionately affects peoples in resource-poor settings, particularly those in Asia and Africa. More than $90 \%$ of new TB cases and deaths occur in developing countries $[1,2]$.

According to the 2011 WHO global TB report, Ethiopia ranks $7^{\text {th }}$ in the list of the world's 22 high burden countries for $\mathrm{TB}$ with estimated incidence rate to be $261 / 100,000$, mortality rate $35 / 100,000$ and the prevalence of all forms TB is estimated to be 394/100,000 [2]. About $40-70 \%$ of HIV patients in Ethiopia are co-

\footnotetext{
* Correspondence: bineymt@gmail.com

'Addis Ababa University, College of Health Science, School of Medical Laboratory Sciences, Addis Ababa, Ethiopia

${ }^{3}$ Addis Ababa University, College of Health Science, School of Public health, Addis Ababa, Ethiopia

Full list of author information is available at the end of the article
}

infected with TB [3]. Recently the country has massively engaged in early case detection, provision of adequate chemotherapy and prevention of transmission to new cases [4]. Despite such efforts, the emergence of drug resistant tuberculosis strains that cannot be cured by standard anti-tuberculosis drug regimens considered as devastating threat to TB control program [5]. Drug resistant tuberculosis commonly arises through the selection of mutated strains by inadequate chemotherapy. Resistance to at least the two major anti-tuberculosis drugs, isoniazid and rifampicin has been termed as multidrug-resistant tuberculosis (MDR-TB). Treatment of MDR-TB requires prolonged and expensive chemotherapy using second line drugs of heightened toxicity and less effective [6,7].

According to WHO Global TB report, Ethiopia is ranked as $15^{\text {th }}$ among 27 high burden $\mathrm{M}(\mathrm{X})$ DR-TB countries. The estimated MDR rate was $(0.9 \%-2.8 \%)$ for 
new cases and $(5.6 \%-21 \%)$ for retreatment cases [2]. Despite the availability of a national estimate, it has been noted that continues evaluation of ongoing treatment program is one of the important aspects in the assessment of TB epidemiology $[8,9]$. Therefore, the present study was to determine the pattern and trend of resistance to first line anti-TB drugs among culture positive retreatment cases at St. Peter's TB Specialized Hospital in Addis Ababa, Ethiopia.

\section{Methods}

\section{Research setting and context}

St. Peter's TB specialized hospital was established in June, 1961 Gregorian Calendar (GC). It is a governmental hospital under Federal Democratic Republic of Ethiopia- Ministry of Health (FMOH). The hospital provides various services especially in tuberculosis diagnosis and treatment. It serves as a referral $\mathrm{TB}$ hospital in Addis Ababa, Ethiopia and has a vision to become Center of Excellence for diagnosis and treatment of TB in East Africa.

\section{Study design and participants}

A Hospital based retrospective study was conducted in Addis Ababa from February 2010 to March 2010 GC to assess the patterns of resistance to anti-TB drugs among previously treated TB patients referred to St. Peter's TB Specialized Hospital. Participants for this study were all previously treated TB cases from January 2004 - December 2008 GC. The study subjects already received the standard first line anti-TB treatment for more than one month but still had positive smears and had to be started on a retreatment regimen (2 S (ERHZ)/1(ERHZ)/5 E3 (RH)3. The hospital provides for those patients refereed to the hospital across the country as the hospital serve as a referral point for tuberculosis management for the country.

\section{Drug susceptibility test}

The drug susceptibility tests were done at Ethiopian Health and Nutrition Research Institute (EHNRI) TB Laboratory. The laboratory used Löwenstein-Jensen (L-J) culture medium on which the specimen was inoculated after decontamination with sodium hydroxide (4\%). Identification of isolates was based on the niacin production test, the nitrate reduction test the para-nitrobenzoic (PNB) acid (500 mg/l) test, and the thiophene-2carboxylic acid hydrazide $(\mathrm{TCH})(2 \mathrm{mg} / \mathrm{l})$ resistance test. Mycobacteriums other than M. tuberculosis complex were excluded from the analysis [10].

Drug susceptibility tests were performed using the simplified variant of the indirect proportion method on L-J medium. The proportion method (Canetti modified) determines the percentage of growth (number of colonies) of defined inoculums on a drug-free control medium versus growth on culture media containing the critical concentration of an anti- tuberculosis drug. The critical drug concentration, as well as the critical proportion of resistant colonies were been evaluated from clinical data. Resistance was expressed as the percentage of colonies that grew on recommended critical concentrations of the drugs tested (i.e. $0.2 \mathrm{mg} / \mathrm{l}$ for isoniazid (INH), $2 \mathrm{mg} / \mathrm{l}$ for ethambutol (EMB), and $4 \mathrm{mg} / \mathrm{l}$ for streptomycin (STM) and $40 \mathrm{mg} / \mathrm{l}$ for rifampicin (RIF). The criterion used for drug resistance was growth of $1 \%$ or more of the bacterial population on media containing the critical concentration of each drug. The results of the tests were recorded on standardized forms [10].

\section{Data collection procedures}

Laboratory based retrospective study was done among TB patients who had retreated for first line antituberculosis drugs. Participant demographic variable, drug susceptibility test results of Mycobacterium tuberculosis (MTB) were carefully collected from the laboratory registration book using a standardized check list. The overall activities of data collection were monitored by the principal investigator and there was strict supervision during data collection. Only those patients with full information recorded in the registration book were included in the study. The extracted variables were age, sex, year of treatment, smear sample, drug resistance. Risk factors for drug resistance could not be evaluated as there was insufficient data in the charts.

\section{Operational definitions}

Resistance in new cases (primary) was defined as in vitro resistance in patients who did not have a history of antiTB treatment, while retreatment resistance (secondary) was defined as in vitro resistance in patients previously treated with any anti-TB medication. The definition of MDR-TB cases recommended by WHO and IUATLD is the pattern of drug resistance to at least INH and RIF. Resistance to more than one agents except these agents was referred to as poly drug resistance TB (PDR-TB) [11].

\section{Data management and analysis}

Data collected in this study were used to ascertain the percentage of drug resistance in each year, to any drug, each drug either alone or in combination with other drugs. The percentage of resistant isolates from patients was calculated by dividing the number of resistant isolates in each of the categories by the total number of isolates tested for that drug or combination of drugs. We evaluated temporal trends in resistance to each drug alone or in combination with other drugs, as well as trends in multidrug resistance. The chi square test for trend was used to assess percentage resistance by year of 
report at the $5 \%$ critical value. Data analysis was conducted using SPSS Version 16.0 software (SPSS INC, Chicago, IL, USA).

\section{Ethical consideration}

Ethical clearance for the conduct of this study was obtained from St. Peter's TB Specialized Hospital Ethical Review Committee and Institutional Ethical Review Committee of Addis Ababa University, Faculty of Medicine; School of Medical Laboratory Science. Names or identification number of study participants were not included from the data collection template.

\section{Findings}

A total of 376 distinct cultures positive retreated cases (previously treated with first line anti- TB drugs for at least one month) cases were identified from January 2004 to December 2008 at St. Peter's TB Specialized Hospital Laboratory registration book. Of these 235 $(62.5 \%)$ were male and the age range of the study population was 9 -74years (Mean age, 31.9), median age of 28 (9-76). Of these $87.5 \%$ was in age range of $15-49$. The study population was $69,83,86,74$ and 64 across per year, respectively (Table 1 ).

Among 376 culture positive for M. tuberculosis one hundred and two $(27.1 \%)$ were susceptible to all of the four first line anti-TB drugs -Isoniazid, Rifampicin, Ethambutol \& Streptomycin, and 274 (72.9\%) were resistance to at least one drug. Resistance to any STM (67.3\%) was found to be the most common followed by (56.1\%) INH, (46.1\%) RIF and (43.5\%) ETB (Figure 1).

Table 1 Socio-demographic characteristics of the study participants, St. Peter's TB Specialized Hospital, 2011

\begin{tabular}{lcc}
\hline Characteristics & Frequency & Percentage (\%) \\
\hline Sex & 235 & 62.5 \\
Male & 141 & 37.5 \\
Female & 376 & 100 \\
$\quad$ Total & & \\
Age & 7 & 1.8 \\
$<15$ Years & 329 & 86.8 \\
15-49 Years & 43 & 11.4 \\
$>49$ Years & 376 & 100 \\
Total & & \\
Years & 69 & 18.2 \\
2004 & 83 & 21.9 \\
2005 & 86 & 22.7 \\
2006 & 74 & 19.5 \\
2007 & 64 & 16.8 \\
2008 & 376 & 100 \\
Total & &
\end{tabular}

Seventy one (18.9\%) were resistant to only a single drug while more than one drug resistance includes both poly resistance and MDR-TB. so that it should be 203 $(203 / 376=54 \%)$ were resistant to two or more drugs. Among mono resistance, Streptomycin 56 (78.8\%) was found to be highest proportion, followed by isoniazid. However, our result showed no mono resistant strain for Rifampicin.

Poly resistance was reported in 29 (7.7\%) of the cases. Among these, the highest proportion was 15(53.6\%) Isoniazid + Streptomycin Combination. In this study the prevalence of MDR-TB (defined as the resistance to at least INH and RIF) 174 (46.3\%) was found to be high (Table 2). Among these, 103 (59.2\%) were males and 154 (90.8\%) were between age range of $15-49$ years. Out of MDR-TB cases 140 (80.5\%) cases were resistant for all first line anti-TB drugs.

Any drug resistance as well as MDR-TB was not statistically significantly associated with any age group. However, there was statistically significant association between any drug resistances among gender, specifically male patients $(\mathrm{P}<0.05)$ (Table 3$)$. Trends in resistance rate among re-treatment cases from 2004 to 2008 showed a statistically significant increase for any drug as well as for INH, RIF, and MDR resistance ( $\mathrm{P}<0.05$ for trend) (Figure 2).

\section{Discussion}

In the present study, 102 (27.1\%) of the cases were susceptible to all of the four first line anti-TB drugs and $274(72.9 \%)$ were resistant to at least one or more antiTB drugs indicating higher prevalence of drug resistant TB for first line anti-TB drugs than studies conducted in Ethiopia with overall resistance to any anti-TB drugs ranges from 14\% -27\% [12-16]. The higher magnitude of resistance to any anti-TB drugs in the present study could be explained the difference in the characteristics study subjects (retreatment cases) which is more likely to have resistance and majority of retreatment cases in the country referred to the study hospital.

Mono resistance to streptomycin (14.9\%) was found to be the highest proportion among first line anti-TB drugs which is consistent with WHO global surveillance report [5] as well as in other studies conducted in Denmark, Turkey, India and Arsi zone of Ethiopia [17-20]. In contrast a 15-year surveillance in Saudi Arabia showed, resistance to INH and EMB was more frequent than to other first-line drugs [21].

MDR-TB in retreatment patients varies from $30 \%$ to $80 \%$ in different regions [22] and in Ethiopia, MDR -TB prevalence in 2008 was $(0.9 \%-2.8 \%)$ for new cases and $(5.6 \%-21 \%)$ for retreatment cases [2].

In this study, MDR-TB was found in $46.3 \%$ of retreatment; this shows a higher percentage of MDR-TB in 


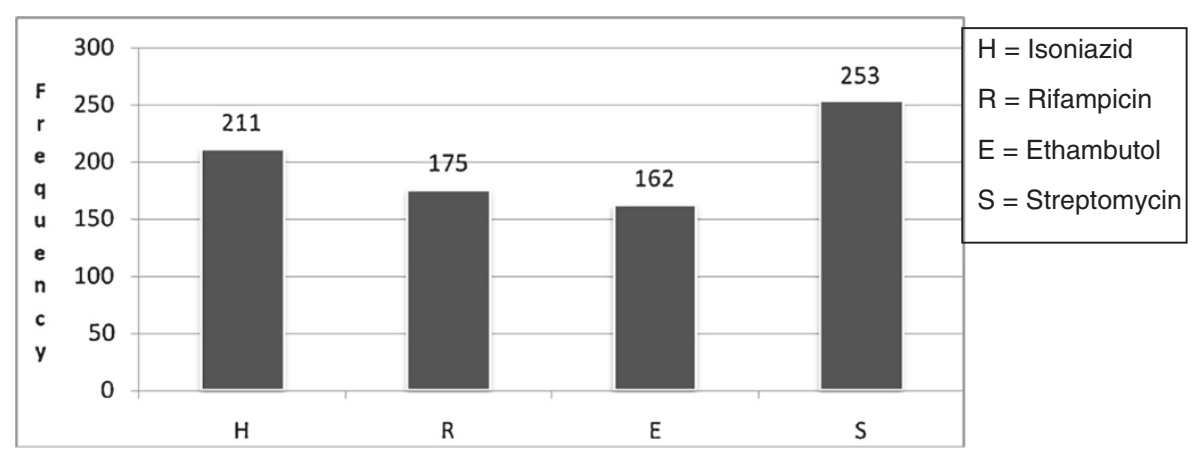

Figure 1 Frequency of First line Tuberculosis Drug resistance in St. Peter's TB Specialized Hospital, 2011.

Ethiopia, particularly in retreatment cases, compared to WHO report [2]. Surprisingly, our study noted that MDR-TB prevalence is significantly higher in male than female patients which are inconsistent with a report by O'Donnell MR et al. where female have more risk than

Table 2 Pattern of drug resistance among 376 cultures positive retreatment cases, St. Peter's TB specialized Hospital, 2011

\begin{tabular}{ccc}
\hline Pattern of drug resistance & Frequency & Percentage (\%) \\
\hline Any drug resistance & 274 & 72.9 \\
INH $^{1}$ & 211 & 56.1 \\
RIF $^{2}$ & 175 & 46.5 \\
ETB $^{3}$ & 162 & 43.1 \\
STM $^{4}$ & 253 & 67.3
\end{tabular}

Single drug

$71 \quad 18.9$

Mono INH Resistant

11

2.9

Mono RIF Resistant

Mono ETB Resistant

Mono STM Resistant

Two drug

$\begin{array}{ccc}\text { INH + RIF } & 4 & 1.1 \\ \text { INH + ETB } & 2 & 0.53 \\ \text { INH + STM } & 15 & 4.0 \\ \text { RIF + ETB } & 0 & 0 \\ \text { RIF + STM } & 0 & 0 \\ \text { ETB + STM } & 0 & 0\end{array}$

Three or more drug

$\begin{array}{ccc}\text { INH + RIF + ETB } & 2 & 0.53 \\ \text { INH + RIF + STM } & 28 & 7.5 \\ \text { INH + ETB + STM } & 12 & 3.2 \\ \mathrm{RIF}+\text { ETB + STM } & 0 & 0 \\ \text { INH + RIF + ETB + STM } & 140 & 37.2 \\ \mathrm{MDR}^{5} & 174 & 46\end{array}$

${ }^{1} \mathrm{INH}=$ Isoniazid, ${ }^{2} \mathrm{RIF}=$ Rifampicin, ${ }^{3} \mathrm{ETB},=$ Ethabutol, ${ }^{4} \mathrm{STM}$, = Streptomycin, ${ }^{5} \mathrm{MDR}=$ Multi drug resistance (resistance at least INH and RIF). male patients [23]. The latter study explained as gender differences in drug-resistant TB in areas of HIV endemicity and low prevalence suggest a possible effect of the HIV/AIDS epidemic on prevalence of drug-resistant TB in women [24]. Despite such difference, male or female TB patients could have different levels of risks for drug resistance due to differences in access to health-care services or exposure to other risk factors. Discovering gender disparities associated with the risks of MDR-TB could provide insight into the development of targeted measures and improve access to health care and reduce the risk of acquiring drug-resistance.

The association between age and the risk of MDR-TB is not well established in the literatures as different studies use different cut-off points for age groups. MDR-TB patients were more likely to be younger than 65 years $[24,25]$. A systematic review analysis in Europe revealed the pooled risk of MDR-TB for people younger than 45 was higher than that among older patients (OR: 1.52, 95\% CI: 1.13-2.03) [24]. In this study, we did not found a statistical significant association between patient's age and frequency of MDR-TB which was in contrast with a report by Shao et al. indicating MDR -TB was much higher in young adulthoods and peaked at 35-44 years old [26].

The trend in drug resistance against all first-line drugs shows a significant increase. This remarkable percentage of drug resistance among retreatment patients supports the idea of inefficiency in TB control programs and irregular/improper anti-TB drug use in recent years, which have led to accumulation and multiplication of resistant strains. Notably, resistance to the other corner stone of anti-TB therapy, RIF mono resistance, was not observed in current study, but there is a significant increase in proportion of INH resistance which may be considered as an alarming sign and indicates further transmission of resistant strains in the community.

The study has its own limitations. There is a potential bias in estimation of drug resistance in previously treated cases and the data were taken from the hospital, it 
Table 3 Characteristics of culture positive retreatment cases according drug resistance pattern, St. Peter's TB Specialized Hospital, 2011

\begin{tabular}{|c|c|c|c|c|c|}
\hline Variable & $\mathrm{N}(\%)$ & Any drug resistance $\mathrm{n}(\%)$ & P-value & MDR-TB n, (\%) & P-value \\
\hline \multicolumn{6}{|l|}{ Sex } \\
\hline Male & $235(62.5)$ & $154(59)$ & 0.03 & $103(59.2)$ & 0.220 \\
\hline Female & $141(37.5)$ & $107(41)$ & & $71(40.8)$ & \\
\hline \multicolumn{6}{|l|}{ Age(yr) } \\
\hline$<15$ & $7(1.9)$ & $5(1.9)$ & 0.990 & $4(2.3)$ & \\
\hline $15-49$ & $330(87.8)$ & $235(90)$ & 0.395 & $158(90.8)$ & 0.689 \\
\hline$>49$ & 39 (10.4) & $21(8)$ & & $12(6.9)$ & 0.333 \\
\hline
\end{tabular}

may be subjected to selection bias, and it was not possible to get other relevant variables such as co-morbidity, HIV status. In resource poor settings where the quality of reports are often questionable and the retrospective data collected primarily for reporting may not be robust [27].

\section{Conclusions}

In conclusion, the high prevalence of drug resistance in this study area may limit the success of TB control program. Increased trend in resistance to anti-TB drug among re-treatment cases were noted. So this signifies that establishing advanced diagnostic facilities for early detection of MDR-TB. Monitoring of drug-resistance should be enhanced by periodic surveys to assess trends and take correct actions when necessary. Prevention and control of drug-resistant TB should be emphasized by the revised DOTS program through prompt case detection, routine and quality-assured DST for those patients at high risk of resistance, proper administration of anti-TB drugs according to the recommended national guidelines and strengthening national DOTS program, expanding second line treatment used for treating the existing cases and help to protect its transmission, This enables to appropriately

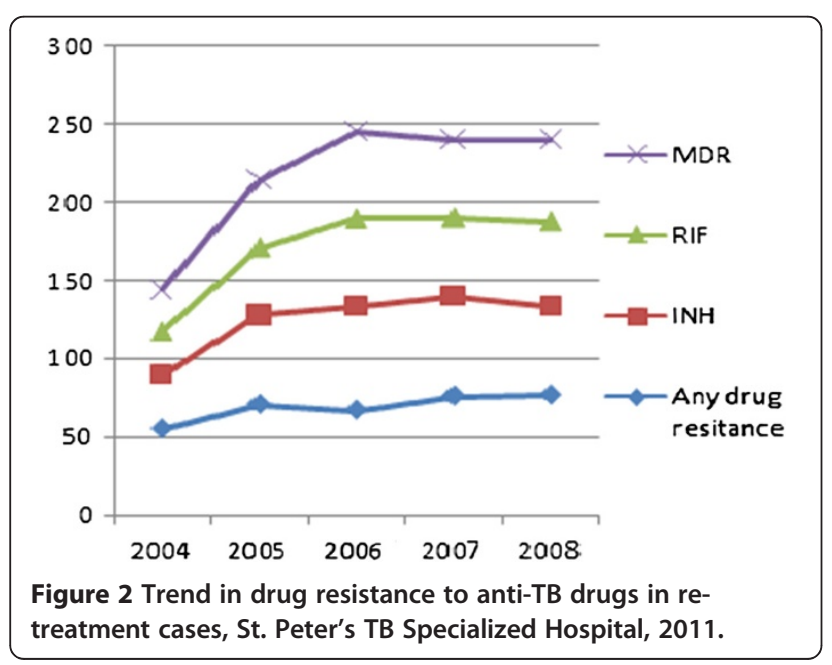

manage TB patients and to lower the rate of MDR-TB among retreatment cases. In addition further studies and continued surveillance of the resistance pattern of $M$. tuberculosis is needed to further delineate the risk factors and to formulate the plans for the future management of tuberculosis in high MDR-TB settings.

\section{Competing interest}

All authors declare that they have no conflict of interest associated with the publication of this manuscript.

\section{Authors' contributions}

DA conceived and designed the study and collected data in the field, performed analysis, Interpretation of data, and draft the manuscript. BT assisted with the design, performed analysis, interpretation of data and the critical review of the manuscript. MA participated in design and performed analysis, interpretation of data, helped in drafting the manuscript and critically reviewed the manuscript. SB participated in interpretation of data, helped in drafting the manuscript and critically reviewed the manuscript. All authors read and approved the final version of the manuscript. All authors participated in critical appraisal and revision of the manuscript.

\section{Acknowledgements}

We would like to be grateful to St. Peter's TB Specialized Hospital staffs for their administrative support and cooperation. Thanks to Dr. Peter Memiah for his assistance with editing of manuscript. The study was funded by Addis Ababa University.

\section{Author details}

${ }^{1}$ Addis Ababa University, College of Health Science, School of Medica Laboratory Sciences, Addis Ababa, Ethiopia. ${ }^{2}$ St. Peter's TB Specialized Hospital, Addis Ababa, Ethiopia. ${ }^{3}$ Addis Ababa University, College of Health Science, School of Public health, Addis Ababa, Ethiopia. ${ }^{4}$ Department of Epidemiology and Biostatistics, Jimma University, College of Public Health and Medical Science, Jimma, Ethiopia.

Received: 10 December 2011 Accepted: 22 August 2012 Published: 28 August 2012

\section{References}

1. WHO: World Health Organization: Global Tuberculosis control: Surveillance, planning and Financing. 2008. http://www.who.int/tb/publications/ global_report/en/] website WHO report.

2. WHO: World Health Organization: Global tuberculosis control: WHO report 2011. 2011. http://www.who.int/about/licensing/copyright_form/en/index. html). WHO report.

3. Demissie MLB, Tegbaru B: Human Immunodeficiency virus (HIV) infection in tuberculosis patients in Addis Ababa. Ethiop J Health Dev 2000, 14:277-282.

4. Federal Ministry of Health: Guideline for program and Clinical Management of drug Resistant Tuberculosis. 1st edition. Addis Ababa, Ethiopia: FMOH; 2009. 
5. WHO: Multidrug and extensively drug-resistant TB (M/XDR-TB): 2010 Global Report on Surveillance and Response. 2010.

6. Wilkinson LSR: Extensively drug resistant tuberculosis. BMJ 2006, 333(7568):559-560.

7. Juan C, Palomino SC, Viviana R: Tuberculosis 2007 from basic science to patient care. $2007: 635-636$.

8. WHO: World Health Organization: Addressing the threat of tuberculosis caused by extensively drug-resistant Mycobacterium tuberculosis. Wkly Epidemiol Rec 2006, 81:386-390.

9. Raviglione MC DC, Schmidt S, Kochi A: Assessment of world-wide tuberculosis control. Lancet 1997, 350:624-629.

10. Ethiopian Health and Nutrition Research Institute: TB Laboratory Standard Operating Procedure (SOP). Addis Ababa, Ethiopia: EHNRI; 2007. Unpublished document.

11. Iseman M: A clinician's guide to tuberculosis. Philadelphia: Lippincott-Williams and Wilkins; 2000:323-353.

12. Meskel DW AG, Lakew $M$, et al: Anti-tuberculosis drug resistance among retreatment patients seen at St Peter. Tubercul Specialized Hosp Ethiop Med J 2008, 46(3):219-225.

13. Mekdes Gebeyehu EL, Getachew E: Prevalence of drug resistant tuberculosis in Arsi Zone. Ethiop Ethiop J Health Dev 2001, 15:11-16.

14. Kassu Desta DA, Eshetu L, Mekdes G, Beniam F: Drug susceptibility of Mycobacterium tuberculosis isolatesfrom smears negative pulmonary tuberculosis patients, Addis Ababa, Ethiopia. Ethiop J Health Dev 2008, 22(2):212-215.

15. Demissie MGM, Berhane Y: Primary resistance to anti-tuberculosis drugs in Addis Ababa, Ethiop. Int J Tuberc Lung Dis 1997, 1(1):64-67.

16. Bruchfeld J, Aderaye G, Palme IB, Bjorvatn B, Ghebremichael S, Hoffner S, Lindquist L: Molecular Epidemiology and Drug Resistance of Mycobacterium tuberculosis Isolates from Ethiopian Pulmonary Tuberculosis Patients with and without Human Immunodeficiency Virus Infection. J Clin Microbiol 2002, 40(5):1636-1643.

17. Thomsen VØ BJ, Lillebaek T, Glisman S: Results from 8 years of susceptibility testing of clinical Mycobacterium tuberculosis isolates in Denmark. Eur Respir J 2000, 16:203-208.

18. Senol GKB, Komurcuoglu A: Drug resistance of Mycobacterium tuberculosis in Western Turkey: a retrospective study from 1100-bed teaching hospital. J Infect 2005, 50(4):306-311.

19. Kart L, Remzi A, Tor M, Gulmez I, Oymak SF, Atmaca HM, Erdem F: Antituberculosis drug resistance patterns in two regions of Turkey: a retrospective analysis. Ann Clin Microbiol Antimicrob 2002, 1:6.

20. Gupta PR, Singhal B, Sharma PN, Gupta RB: Prevalence of initial drug resistance in tuberculosis patients attending a chest hospital. Ind J Med Res 1993, 97:102-103.

21. JA A-MA A-Tq, Abed MS: Susceptibility pattern and epidemiology of Mycobacterium tuberculosis in a Saudi Arabian hospital: a 15-year study from 1989 to 2003. Chest 2005, 128:3229-3232.

22. Pablos Me'ndez ARM, Laszlo A, Binkin N, Rieder HL, Bustreo F, et al: Global surveillance for anti-tuberculosis drug resistance,1994-1997. N Engl J Med 1998, 338:1641-1649.

23. O'Donnell MR, Zelnick J, Werner L, Master I, Loveday M, Horsburgh CR, Padayatchi N: Extensively drug-resistant tuberculosis in women, KwaZulu-Natal, South Africa. Emerg Infect Dis 2011, 17(10):1942-1945.

24. Faustini AHA, Perucci CA: Risk factors for multidrug resistant tuberculosis in Europe: a systematic review. Thorax 2006, 61:158-163.

25. Law WS YW, Chiu C, Kam KM, Tam CM, Chan CK, Leung CC: Risk factors for multidrug-resistant tuberculosis in Hong Kong. Int J Tuberc Lung Dis 2008, 12:1065-1070.

26. XW SYYD, Lu W, Song H, Dai Y, Shen H, Wang J: Epidemiology of anti-tuberculosis drug resistance in a chinese population: current situation and challenges ahead. BMC Public Health 2011, 11:110.

27. WHO: Tuberculosis control program Resolution WHA44.8. Forty-fourth World Health Assembly. In Handbook of resolutions and decisions of the World Health Assembly and the Executive Board. Volume III, 3rd edition (1985-1992). Geneva: WHO; WHO document WHA44/1991/REC/1; 1993

doi:10.1186/1756-0500-5-462

Cite this article as: Abate et al.: Epidemiology of anti-tuberculosis drug resistance patterns and trends in tuberculosis referral hospital in Addis Ababa, Ethiopia. BMC Research Notes 2012 5:462.

\section{Submit your next manuscript to BioMed Central and take full advantage of:}

- Convenient online submission

- Thorough peer review

- No space constraints or color figure charges

- Immediate publication on acceptance

- Inclusion in PubMed, CAS, Scopus and Google Scholar

- Research which is freely available for redistribution 